\title{
Isolation and Characterization of Photosynthetic Bacteria from Municipal Waste
}

\author{
Malik Sajad Ahmad ${ }^{1}$, M.Y. Zargar ${ }^{2}$, S.A. Mir ${ }^{4}$, N.A. Bhat ${ }^{3}$, Z.A. Baba ${ }^{1}$, Rehana Habib \\ Kant $^{5}$, Zaffar M. Dar ${ }^{1}$, Imtiyaz Jahangir Khan ${ }^{1}$ and Jigmet Yangchan ${ }^{6}$ \\ ${ }^{1}$ Division of Basic Sciences and Humanities, ${ }^{2}$ Directorate of Research, ${ }^{3}$ Division of Plant \\ Pathology, ${ }^{4}$ Division of Agri. Statistics, ${ }^{5}$ Division of Agronomy, Faculty of Agriculture, \\ ${ }^{6}$ HMAARI Leh, SKUAST-K, Shalimar, Srinagar, 190025 \\ *Corresponding author
}

Keywords

Anaerobic-light, benzoate,

Chlorobium sp.,

Gram- negative,

Rhodoferax sp.,

Rhodopseudomonas

sp., succinate

Article Info

Accepted:

10 February 2019

Available Online:

10 March 2019

\section{A B S T R A C T}

One of the photosynthetic bacterial isolate $\mathrm{L} 6$ grown at $10{ }^{\circ} \mathrm{C}$ confirmed as Rhodoferax sp. and six isolates showed growth at $30{ }^{\circ} \mathrm{C}$ were isolated from municipal wastes in Kashmir and Ladakh valleys. The cells were Gram-negative, pigmented, non-spore-forming, mostly oxidase positive except K2 isolate and nitrate reduction negative except G17 isolate. All isolates showed growth in aerobic-light conditions except $\mathrm{K} 2$ and anaerobic-light except L6. All isolates utilized acetate, malate and succinate as carbon source while as benzoate and mannitol were not used except G17. However mannitol was used as carbon source by L6 also. Isolate $\mathrm{K} 2$ had shown well defined growth in sulphide medium and was regarded as sulphur bacterium while as isolates G17, L6, L27, K5, S5 and S7 were unable to utilize sulphide and thiosulphate as electron donor and considered as non sulphur bacteria. The morpho-biochemical test further confirmed K2 isolate as Chlorobium sp., S5 and L27 as Rhodospirillum sp., S7 as Rhodobacter sp., G17 as Rhodopseudomonas sp., K5 as Rhodomicrobium sp. Isolates G17, L6, L27, S5 and S7 were observed the most efficient on the basis of versatility in carbon utilization character. Isolate L6 was regarded as the psychrophillic/psychrotolerant photosynthetic purple non sulphur bacterium.

\section{Introduction}

In Jammu \& Kashmir, only 1\% of 1374 MT of municipal solid waste generated per day gets processed (Ministry of Housing and Urban Affairs, GOI, 2018). Waste generation and its control have taken an important role in our environment. With the doubling of population and changing lifestyle pattern of the inhabitants the quantity of municipal waste generated is increasing in an alarming rate. Municipal solid waste is made up of different organic and inorganic fractions like food, vegetables, paper, wood, plastics, glass, metal and other inert material (Mor, 2006). The waste sites harbour many types of microorganisms beneficial as well as deleterious. Photosynthetic bacteria are 
ubiquitous in nature. They are present in aquatic environments and form water blooms. The presence of purple non sulphur bacteria is particularly dependent upon the degree to which water is polluted by organic matter. Their growth contributes to the purification of the heavily polluted water exposed to sunlight e.g, in sewage lagoons (Holm and Vennes, 1970). The photosynthetic bacteria are used in the main purification stage of organic waste water treatment (Kobayashi, 1975). Approaches to understand the types of photosynthetic bacteria growing in the municipal waste, the samples were collected from various sites of Kashmir and Ladakh valleys. The aim of the study was the isolation and characterization of photosynthetic bacteria from these waste samples based on the morphological and biochemical characteristics.

\section{Materials and Methods}

Four survey area viz. Gulmarg, Srinagar, Leh and Kargil were selected and from each area two sites were selected. Four dumpy samples of biodegradable wastes which had access to sunlight containing a substantial pigmented growth (soil mixed with waste and water) were collected from four different locations of each site and then composited into one sample per site. So, eight composite samples were collected in sterile zip-lock plastic bags maintaining aseptic conditions from eight waste rich sites, marked according to their source and site and stored at $4{ }^{\circ} \mathrm{C}$. Purposive Method of Sampling was adopted during the collection of samples and the location wise details are furnished in the Table 1.

The dumpy waste samples collected from sites were grown anaerobically under light source of 3000 to 5000 lux in the sterile bottles containing Pfennig's medium (Pfennig Norbert, 1967) for enrichment of purple nonsulfur photosynthetic bacterial colonies till they form "bloom". Once the enrichment had achieved turbidity with the consequent bloom, it was streaked onto plates which were then incubated anaerobically near the light source at $10^{\circ} \mathrm{C}$. The initial $\mathrm{pH}$ of the medium was adjusted to $\mathrm{pH} 7.0$ using $5 \mathrm{M} \mathrm{NaOH}$ which was raised to 9.0 on subsequent streaking methods. The isolates were purified by repeated streaking on plates and the pure cultures were kept on agar slants at $4{ }^{\circ} \mathrm{C}$ for further use. Also, the growth of the photosynthetic bacteria in the samples was enhanced by incubation under anaerobic conditions and 800 Lux ('anaerobic-light') at room temperature $\left(30{ }^{\circ} \mathrm{C}\right)$ for 4 days. One gram of the incubated sample was diluted in $100 \mathrm{ml}$ of distilled water and plated on Pringsheim's media run in the triplicates and incubated for a week. The clear colonies formed were enriched in G5 medium (Kohlmiler and Gest, 1951). A loopful of pinkish culture broth was streaked onto G5 agar without vitamins. After 7 days' incubation, each colony was picked up and streaked onto new G5 agar plates. This procedure was repeated (on sulfide medium also) until pure cultures were obtained. Sodium succinate or malate was used as an organic carbon source for isolation of purple non- sulphur bacteria because these are not generally used by other bacteria under photoheterotrophic growth conditions with no hydrogen sulfide.

The isolates were identified according to the procedures described by Watanabe et al. (1981). The stock cultures of the isolates were stored at $4^{\circ} \mathrm{C}$ overlying with sterile liquid paraffin under anaerobic light condition for 23 months. The isolated photosynthetic bacteria were characterized on the basis of colony morphology, colour, growth characteristics, Gram staining, spore forming ability, mobility, catalase, oxidase, urease, nitrate reduction and carbohydrate utilization tests (Masanobu, 2003). The compositions of 
selective media used for the isolation of photosynthetic bacteria are given in Table 2 .

\section{Results and Discussion}

Six isolates of non-sulphur and an isolate of sulphur photosynthetic bacteria were purified, characterized and stored for further use. Isolate $\mathrm{K} 2$ had shown well defined growth in sulphide medium and was regarded as sulphur bacterium while as isolates G17, L6, L27, K5, S5 and S7 were unable to utilize sulphide and thiosulphate as electron donor and considered as non sulphur bacteria.
The morpho-biochemical test further confirmed K2 isolate as Chlorobium sp., S5 and L27 as Rhodospirillum sp., S7 as Rhodobacter sp., G17 as Rhodopseudomonas sp., K5 as Rhodomicrobium sp. and L6 has shown growth at $10{ }^{\circ} \mathrm{C}$ confirmed as Rhodoferax sp. Isolates G17, L6, L27, S5 and S7 were observed the most efficient on the basis of versatility in carbon utilization character. Isolate L6 was regarded as the psychrophillic/psychrotolerant photosynthetic purple non sulphur bacterium. The results are presented in Table 3 and 4.

Table.1 Location of samples collected from Kashmir and Ladakh for analysis

\begin{tabular}{|c|c|c|c|}
\hline S.No. & Survey area & Site & Sample Site Coordinates \\
\hline 1. & \multirow[t]{2}{*}{ Gulmarg } & Near Hotel Hill Top & $34^{\circ} 2^{\prime} 46.57^{\prime \prime} \mathrm{N} 74^{\circ} 23^{\prime} 15.82^{\prime \prime} \mathrm{E}$ \\
\hline 2. & & Gulmarg Meadow & $34^{\circ} 4^{\prime} 9.08^{\prime /} \mathrm{N} 74^{\circ} 22^{\prime} 24.93^{\prime /} \mathrm{E}$ \\
\hline 3. & \multirow[t]{2}{*}{ Srinagar } & Dargah Hazratbal & $34^{\circ} 7^{\prime} 38.16^{\prime \prime} \mathrm{N} 74^{\circ} 50^{\prime} 18.06^{\prime \prime} \mathrm{E}$ \\
\hline 4. & & Achan Landfill Area & $34^{\circ} 9^{\prime} 18.41^{\prime \prime} \mathrm{N} 74^{\circ} 49^{\prime} 0.20^{\prime / E}$ \\
\hline 5. & \multirow[t]{2}{*}{ Leh } & HMAARI & $33^{\circ} 58^{\prime} 46.13^{\prime \prime} \mathrm{N} 77^{\circ} 41^{\prime} 56.03^{\prime \prime} \mathrm{E}$ \\
\hline 6. & & Leh Golf Course & $34^{\circ} 8^{\prime} 23.26^{\prime \prime} \mathrm{N} 77^{\circ} 34^{\prime} 50.05^{\prime \prime} \mathrm{E}$ \\
\hline 7. & \multirow[t]{2}{*}{ Kargil } & KVK & $34^{\circ} 32^{\prime} 17.13^{\prime \prime} \mathrm{N} 76^{\circ} 09^{\prime} 02.38^{\prime \prime} \mathrm{E}$ \\
\hline \multirow[t]{2}{*}{8.} & & New Sabzi Mandi & $34^{\circ} 33^{\prime} 37.03^{\prime \prime} \mathrm{N} 76^{\circ} 7^{\prime} 33.03^{\prime \prime} \mathrm{E}$ \\
\hline & \multicolumn{3}{|c|}{ Total Composite Sample $=08$} \\
\hline
\end{tabular}

Table.2 Composition of media used for isolation of Photosynthetic bacteria

\begin{tabular}{|c|c|c|}
\hline S.No. & Media used & Composition/ litre of Distilled $\mathrm{H}_{2} \mathrm{O}$ \\
\hline 1. & $\begin{array}{l}\text { Pringsheim's } \\
\text { Medium }\end{array}$ & $\begin{array}{l}\mathrm{K} \mathrm{NO}_{3}=0.2 \mathrm{~g} ; \mathrm{Mg} \mathrm{SO}{ }_{4} \cdot 7 \mathrm{H}_{2} \mathrm{O}=0.01 \mathrm{~g} \\
\left(\mathrm{NH}_{4}\right)_{2} \mathrm{HPO}_{4}=0.02 \mathrm{~g} ; \mathrm{CaCl}_{2} \cdot 6 \mathrm{H}_{2} \mathrm{O}=0.005 \mathrm{~g} ; \mathrm{FeCl}_{3}=0.0005 \mathrm{~g}\end{array}$ \\
\hline 2. & $\begin{array}{l}\text { Pfennig's } \\
\text { medium }\end{array}$ & $\begin{array}{l}\mathrm{KH}_{2} \mathrm{PO}_{4}=0.33 \mathrm{~g} ; \mathrm{MgSO}_{4} .7 \mathrm{H}_{2} \mathrm{O}=0.33 \mathrm{~g} ; \mathrm{NaCl}=0.33 \mathrm{~g} ; \mathrm{NH}_{4} \mathrm{Cl}=0.5 \mathrm{~g} ; \\
\mathrm{CaCl}_{2} .2 \mathrm{H}_{2} \mathrm{O}=0.05 \mathrm{~g} ; \text { Sodium succinate }=1 \mathrm{~g} ; \text { Yeast extract }=0.02 \mathrm{~g} . \mathrm{pH} \\
6.8-7.2 \mathrm{After} \text { autoclaving} \text {, sterile solutions of Trace salts solution }=1.0 \mathrm{ml} \\
\text { and } 0.02 \% \mathrm{FeSO}_{4} .7 \mathrm{H}_{2} \mathrm{O} \text { solution }=0.5 \mathrm{ml} \text { were added. Trace Salt Solution } \\
\left(\mathrm{ZnSO}_{4} .7 \mathrm{H}_{2} \mathrm{O}=10 \mathrm{mg} ; \mathrm{MnCl}_{2} .4 \mathrm{H}_{2} \mathrm{O}=3 \mathrm{mg} ; \mathrm{H}_{3} \mathrm{BO}_{3}=30 \mathrm{mg} ; \mathrm{CoCl}_{2} .6 \mathrm{H}_{2} \mathrm{O}\right. \\
=20 \mathrm{mg} ; \mathrm{CuCl} 2.2 \mathrm{H}_{2} \mathrm{O}=1 \mathrm{mg} ; \mathrm{NiCl}_{2} .6 \mathrm{H}_{2} \mathrm{O}=2 \mathrm{mg} ; \mathrm{Na}_{2} \mathrm{MoO}_{4}=3 \mathrm{mg} ; \\
\left.\text { Distilled } \mathrm{H}_{2} \mathrm{O}=1000 \mathrm{ml} . \mathrm{pH}=3-4\right) . \text { For solid medium preparation Sodium } \\
\text { succinate }=1 \mathrm{~g} ; \text { Yeast extract }=1 \mathrm{~g} \text { and Agar }=15 \mathrm{~g} \text { were additionally added } \\
\text { to the medium. }\end{array}$ \\
\hline 3. & G5 medium & $\begin{array}{l}\text { Peptone }=5 \mathrm{~g} ; \text { Yeast extract }=5 \mathrm{~g} ; \mathrm{L}-\text { glutamic acid }=4 \mathrm{~g} ; \text { Malic acid }=3.5 \\
\mathrm{~g} ; \mathrm{KH}_{2} \mathrm{PO}_{4}=0.12 \mathrm{~g} ; \mathrm{K}_{2} \mathrm{HPO}_{4}=0.18 \mathrm{~g} ; \text { Agar }=15 \mathrm{~g} . \text { Initial } \mathrm{pH}=7.0 \text { by } \\
\text { using } 5 \mathrm{M} \mathrm{NaOH}\end{array}$ \\
\hline 4. & $\begin{array}{l}\text { Sulfide } \\
\text { medium }\end{array}$ & $\begin{array}{l}\mathrm{Na}_{2} \mathrm{~S}=0.1 \mathrm{~g} ; \mathrm{Na}_{2} \mathrm{HCO}_{3}=0.02 \mathrm{~g} ;\left(\mathrm{NH}_{4}\right)_{2} \mathrm{SO}_{4}=0.132 \mathrm{~g} ; \text { Basal medium }( \\
\text { Nicotinic acid }=1.0 \mathrm{mg} ; p \text {-aminobanzoic acid }=1.0 \mathrm{mg} ; \text { thiamine }=1.0 \\
\text { mg; biotin }=0.001 \mathrm{mg}) \text { Distilled } \mathrm{H}_{2} \mathrm{O}=100 \mathrm{ml} . \mathrm{pH}=6.8\end{array}$ \\
\hline
\end{tabular}


Table.3 Differentiating characteristics of photosynthetic bacterial isolates

\begin{tabular}{|c|c|c|c|c|c|c|c|}
\hline Characteristics & G17 & L6 & L27 & K2 & K5 & S5 & S7 \\
\hline Growth Temp. $\left({ }^{\circ} \mathrm{C}\right)$ & 30 & 10 & 30 & 30 & 30 & 30 & 30 \\
\hline Aerobic-light & + & + & + & - & + & + & + \\
\hline Aerobic-dark & + & + & + & - & - & + & + \\
\hline Anaerobic-light & + & - & + & + & + & + & + \\
\hline Anaerobic-dark & - & + & + & - & - & + & + \\
\hline Growth at Ph & 7.2 & 6.5 & 7.2 & 6.0 & 6.8 & 7.2 & 7.2 \\
\hline Colony Morphology & $\begin{array}{l}\text { Orange, } \\
\text { large, } \\
\text { mucoid }\end{array}$ & $\begin{array}{l}\text { Brown, Smooth, } \\
\text { circular \& } \\
\text { convex }\end{array}$ & Purple, & Green, & $\begin{array}{l}\text { Red, tiny, } \\
\text { rough, hard }\end{array}$ & Purple, & Red, small \\
\hline Cell shape & $\begin{array}{l}\text { curved, } \\
\text { knobby } \\
\text { rods }\end{array}$ & Curved rods & $\begin{array}{l}\text { Curved } \\
\text { rods }\end{array}$ & $\begin{array}{l}\text { Long } \\
\text { chains of } \\
\text { cocci }\end{array}$ & $\begin{array}{l}\text { oval cells } \\
\text { connected by } \\
\text { filaments }\end{array}$ & $\begin{array}{l}\text { Curved } \\
\text { rods }\end{array}$ & Spherical \\
\hline Mobility & Motile & Motile & motile & Motile & Non motile & Motile & Motile \\
\hline Gram staining & - & - & - & - & - & - & - \\
\hline Spore forming ability & Absent & Absent & Absent & Absent & Absent & Absent & Absent \\
\hline
\end{tabular}

Positive/Utilization: (+) ; Negative/Non Utilization : (-)

Table.4 Differentiating characteristics of photosynthetic bacterial isolates

\begin{tabular}{|c|c|c|c|c|c|c|c|}
\hline $\begin{array}{l}\text { Characteristics/(Carbohydra } \\
\text { te fermentation) }\end{array}$ & G17 & L6 & L27 & K2 & K5 & S5 & S7 \\
\hline Catalase test & + & - & + & - & + & + & + \\
\hline Oxidase test & + & + & + & - & + & + & + \\
\hline Urease test & + & - & + & - & + & + & + \\
\hline Motility test & + & + & + & + & - & + & + \\
\hline Nitrate Reduction test & + & - & - & - & - & - & - \\
\hline Acetate & + & + & + & + & + & + & + \\
\hline Benzoate & + & - & - & - & - & - & - \\
\hline Citrate & + & - & + & - & - & + & + \\
\hline Ethanol & + & + & + & - & + & - &.- \\
\hline D-fructose & + & + & + & + & - & + & - \\
\hline D-Glucose & + & + & + & - & - & + & + \\
\hline Glutamate & + & - & + & - & $\mathrm{v}$ & + & + \\
\hline Glycerol & + & + & + & - & + & - & - \\
\hline Malate & + & + & + & + & + & + & + \\
\hline Maltose & + & - & + & - & - & + & + \\
\hline Mannitol & + & + & - & - & - & - & - \\
\hline Succinate & + & + & + & + & + & + & + \\
\hline Sulphide & - & - & - & + & - & - & - \\
\hline Thiosulphate & - & - & - & + & - & - & - \\
\hline Probable genus & $\begin{array}{l}\text { Rhodopseudo } \\
\text { monas sp. }\end{array}$ & $\begin{array}{l}\text { Rhodoferax } \\
\text { fermentans }\end{array}$ & $\begin{array}{l}\text { Rhodospiri } \\
\text { llum sp. }\end{array}$ & $\begin{array}{c}\text { Chlorobium } \\
\text { sp. }\end{array}$ & $\begin{array}{l}\text { Phodomicro } \\
\text { bium sp. }\end{array}$ & $\begin{array}{l}\text { Phodospiri } \\
\text { llum sp. }\end{array}$ & $\begin{array}{l}\text { Rhodobact } \\
\text { er sp. }\end{array}$ \\
\hline
\end{tabular}

Positive/Utilization: (+) ; Negative/Non Utilization : (-); Variable : (v)

In looking for the purple non-sulfur bacteria, sodium succinate or malate was used as an organic carbon source because these are not generally used by other bacteria under 
photoheterotrophic growth conditions with no hydrogen sulfide.

In conclusion, seven different photosynthetic bacteria were isolated from the eight composite samples of municipal wastes collected from Ladakh and Kashmir divisions of Himalaya range. Out of them one isolate L6 was psychrophilic/ psychrotolerant photosynthetic purple non sulphur bacterium. The colonies of L6 were brown, smooth, circular and convex; Gram-negative, curved rods, motile, non-sporeforming cells; catalase, urease and nitrate reduction negative. All isolates showed growth in aerobic-light conditions except $\mathrm{K} 2$ and anaerobic-light except L6. All isolates utilized acetate, malate and succinate as carbon source. Isolate $\mathrm{K} 2$ had shown well defined growth in sulphide and thiosulphate media and was regarded as sulphur bacterium while as isolates G17, L6, L27, K5, S5 and S7 were unable to utilize sulphide and thiosulphate as electron donor and considered as non sulphur bacteria. The isolate L6 was confirmed as Rhodoferax sp., K2 isolate as Chlorobium sp., S5 and L27 as Rhodospirillum sp., S7 as Rhodobacter sp., G17 as Rhodopseudomonas sp., K5 as Rhodomicrobium sp. on the basis of varied morpho-biochemical characters. Isolates G17, L6, L27, S5 and S7 were observed the most efficient on the basis of versatility in carbon utilization character.

\section{Acknowledgement}

The authors are thankful to the Hon'ble Vice Chancellor and Director Research, Sher-eKashmir University of Agricultural Sciences \& Technology of Kashmir for providing technical and research facilities.

\section{References}

Holm, H.W. and Vennes, I.W.1970. Occurance of purple sulphur bacteria in sewage treatment lagoon. Appl. Microbiol.19: 988-996.

Kobayashi M.1975.

Role of photosynthetic bacteria in foul $\mathrm{w}$ ater purification. Prog. Water Technol. 7: 309- 315.

Kohlmiler, E. F. and Gest, H.1951. A comparative study fermentation of organic acids by Rhodospirillum rubrum. J. Bacteriol. 61: 296-282.

Masanobu, S. 2003. Co-Existance of Photosynthetic Bacteria, Streptomyces and lactic acid bacteria in solutions of effective microorganisms. EM Laboratory, International Nature farming Research Center, Japan, pp. 22.

Ministry of Housing and Urban Affairs. 2018. Government of India. Retrieved from http://envfor.nic.in/content/draftmunicipal-waste-management-state-wisewaste-generation-2018-comments-invited

Mor, S., Ravindra, K., Dahiya, R. P. and Chandra, A. 2006. Leachate characterization and assessment of groundwater pollution near municipal solid waste landfill site. Environmental Monitoring and Assessment, 118:435456.

Pfennig Norbert, 1967. Photosynthetic bacteria. Annu. Rev. Microbiol. 21:285-324.

Watanabe, K., Kim, J.S., Ito, K., Buranakarl, L., Kampee, T. and Takahashi, H. 1981. Thermostable Nature of Hydrogen Production by Non-Sulfur Purple Photosynthetic Bacteria Isolated in Thailand. Agric. Biol. Chem. 45(1) : 217222.

\section{How to cite this article:}

Malik Sajad Ahmad, M.Y. Zargar, S.A. Mir, N.A. Bhat, Z.A. Baba, Rehana Habib Kant, Zaffar M. Dar, Imtiyaz Jahangir Khan and Jigmet Yangchan. 2019. Isolation and Characterization of Photosynthetic bacteria from Municipal Waste. Int.J.Curr.Microbiol.App.Sci. 8(03): 861-865. doi: https://doi.org/10.20546/ijcmas.2019.803.103 